\title{
Juvenile Systemic Sclerosis
}

National Cancer Institute

\section{Source}

National Cancer Institute. Juvenile Systemic Sclerosis. NCI Thesaurus. Code C117103.

Systemic sclerosis that is diagnosed in children. Juvenile systemic sclerosis is more likely to be of the overlap variant and presents with musculoskeletal involvement. 